\title{
AT-tributable risks?
}

\section{Timothy Bishop ${ }^{1}$ and John Hopper ${ }^{2}$}

\section{'ICRF Genetic Epidemiology Laboratory, Ashley Wing, St. James's University Hospital, Beckett Street, Leeds LS8 2QG, UK. ${ }^{2}$ Department of Public Health and Community Medicine, University of Melbourne, 200 Berkeley Street, Melbourne, Australia}

Genes and breast cancer risk have been a consistent theme in the genetics literature over the last seven years, with the major focus on two genes, BRCA1 and BRCA2, which are associated with a particularly high risk of breast cancer. The excitement generated during the mapping and cloning of these genes is as well known to the general public as to those involved. A review of these publications - more specifically a review of the Discussion sections - shows a consistent theme: from early on, the indication that these genes accounted for only a few percent of all breast cancer was taken on board by most (but certainly not all) authors. Thus, the Discussion usually contained text saying something like "...while $B R C A 1$ and $B R C A 2$ may explain only $1-2 \%$ of breast cancer, common, lower penetrance genes could explain a greater proportion." One candidate for these other genes has been the gene for AT. AT is of course the abbreviation for ataxia telangiectasia, an autosomal recessive syndrome characterized by progressive cerebellar ataxia and oculocutaneous telangiectasia. Individuals homozygous for a mutation in the AT gene (ATM) develop cancer at a rate of 100 times that of the general population and have an increased risk of breast cancer; speculation has been that female heterozygotes also have an increased risk of breast cancer ${ }^{1}$, making AT heterozygosity the clearest candidate for being a common predisposing condition. Two publications have now appeared that estimate the risk of breast cancer in female AT heterozygotes and come up with apparently discrepant results: one by Athma and colleagues finds evidence of an increased risk (estimated at four times the population rate $)^{2}$, the other, by Fitzgerald and colleagues in this issue of Nature Genetics, finds no evidence of an increased risk at all ${ }^{3}$.

The study by Athma et al. involves 'following' identified AT mutations through the families of those with clinically recognized $\mathrm{AT}^{2}$. Each parent of an AT case, of course, carries a mutation and analysis of DNA markers flanking the AT gene allow the authors to identify precisely which female relatives with breast cancer carry an AT mutation. On the basis of the genetic relationship between each case and the AT proband, the a priori probability that these two should share the AT mutation can be calculated. This leads to an estimated relative risk of 3.8 as compared to non-carri- ers. This result is similar to that found by Easton ${ }^{4}$ who reanalysed the previous studies of breast cancer risk in mothers (and other close relatives) of AT cases.

In the publication of FitzGerald et al. ${ }^{3}$, $0.5 \%$ ( 2 of 402 ) breast cancer cases diagnosed under the age of 40 years were AT heterozygotes as compared to $1 \%$ (2 of 202) of controls. Thus, this study shows no evidence of an increased risk for breast cancer for AT heterozygotes. The natural question to ask is 'Why the difference between the studies?' But, in fact, the more relevant question is 'Is there any difference between the studies?' In this regard, several points need to be made before thinking about explanations:

Confidence. In epidemiology, the estimate of the relative risk is qualified by the $95 \%$ confidence interval (CI). The $\mathrm{CI}$ is the estimated range of the true relative risk that is consistent with the observed data. The smaller the size of the $\mathrm{Cl}$, the greater the precision of the estimate; the larger, the less certain. Thus, on the basis of only four AT heterozygotes in 604 cases and blooddonor controls, the $\mathrm{CI}$ from the FitzGerald et al. study reaches up to a seven-fold increased risk. That is, the data are not only consistent with no increased risk in heterozgotes, they are also consistent with any effect up to a seven-fold increased risk! For the Athma et al. study, the $\mathrm{CI}$ is from 1.7 to 8.4 , so that again there is less certainty in the estimate, but because the range does not include 1.0 , it does show significant evidence of an increased risk. We can only conclude that although the FitzGerald et al. study found no evidence of an increased risk, it is still consistent with the results of Athma et al. Unfortunately, when relying on such a small numbers of mutation carriers in the FitzGerald et al. study or on such a small number of breast cancers in the Athma et al. study, chance is a major factor and, as a result, the precision of the estimate suffers.

Carrier frequency. The large size of the $\mathrm{CI}$ from the FitzGerald et al. study results primarily from the lack of knowledge of the AT carrier frequency; the above analysis assumes that it is unknown and must be estimated from their study. If it were known exactly for the Boston population studied, then their estimated relative risk would be more precise. The carrier frequency in the general population is not an issue for the Athma et al. study because they start off from AT homozygotes.

Study size. FitzGerald et al. quote, quite correctly, that if the population frequency of AT heterozygotes is $1 \%$ then their study has $90 \%$ power to detect a six-fold increased risk. This means that if female AT heterozygotes had a six-fold increased risk of breast cancer, then they were extremely likely to detect such an effect. However, a study even of the magnitude of this study has only a two in five chance (that is, $40 \%$ power) to detect an increased risk of fourfold, $20 \%$ power for three-fold and $6 \%$ power to detect a two-fold increased risk.

Study Design. These two study designs are quite complementary. The Athma et al. study design provides an efficient scheme to estimate the relative risk of breast cancer in AT heterozygotes but provides no information with regard to the prevalence of AT heterozygotes in the general population (and hence the proportion of breast cancer due to AT heterozygosity - the attributed risk). The Fitzgerald et al. study gives limited information about the relative risk but provides an estimate of the prevalence of AT heterozygotes; indeed the upper limit of the $95 \% \mathrm{Cl}$ for the proportion of early onset breast cancer occurring at AT heterozygotes is $2.4 \%$ (assuming that their assay identified $75 \%$ of all mutations).

Finally, conclusions from epidemiological studies come from identifying consistencies from a number of studies. Neither study discussed here reveals the magnitude of the risk unequivocally, but this situation is not unique and is in fact remarkably similar to the state of knowledge regarding the penetrance of $B R C A 1$ and $B R C A 2$ in mutation carriers. While there are scientific and academic reasons for gaining this knowledge, the clinical needs override these. The authors of both studies must be congratulated for their efforts because only with such detailed studies will the answers eventually become clear. We are certainly not at that stage yet and must await the results of large-scale population-based studies which pay particular attention to the selection of cases and matched controls.

1. Swift, M., Sheiman, L. \& Perry, M. Cancer Res. 36 , 209-215 (1976)

2. Athma, P., Rappaport, R. \& Swift, M. Cancer Genet. Cytogenet. 92, 130-134 (1996).

3. FitzGerald, M.G. et al. Nature Genet. 15, 307-310 (1997).

4. Easton, D. Int. J. Radiat. Biol. 66, 5177-182 (1994). 